\title{
GESTIÓN DINÁMICA DE LA ACCIÓN TUTORIAL EN LA UNIVERSIDAD DE CÓRDOBA
}

\section{DYNAMIC MANAGEMENT OF OFFICE HOURS IN THE UNIVERSITY OF CÓRDOBA}

\author{
Tomás Morares de Luna1, Alma L. Albujer Brotons², José Garres Díaz³ \& \\ Jónatan Herrera Fernández 4
}

Fecha de recepción: 14/12/2020; Fecha de revisión: 19/01/2020; Fecha de aceptación: 12/02/2021.

Cómo citar este artículo:

Morales de Luna, T., Albujer Brotons, A. L., Garres Díaz, J. \& Herrera Fernández, J. (2021). Gestión dinámica de la acción tutorial en la Universidad de Córdoba. Revista de Innovación y Buenas Prácticas Docentes, 10(1), 71-86.

\section{Autor de Correspondencia:Tomas.Morales@uco.es}

\begin{abstract}
Resumen
En este trabajo se analiza el uso de herramientas para la planificación y reserva de tutorías, así como para la realización de las mismas de forma virtual por parte de los estudiantes de diversos grados de la Universidad de Córdoba a lo largo del curso 2019/2020. Estas herramientas permiten, tanto al alumnado como al profesorado, un mayor aprovechamiento de su tiempo y una gestión dinámica de la acción tuto rial. Además, su aplicación resultó al final esencial por la situación generada por la COVID-19, siendo necesario la virtualización, no solo de las tutorías, sino también de toda la docencia. Tras finalizar el curso 2019/2020, en el cual se tenía prevista esta experiencia, se ha encuestado al estudiantado sobre las herramientas utilizadas. Se ha obtenido una valoración muy satisfactoria de su uso, concluyendo que no sólo facilita el acceso a tutorías, sino que se trata de un sistema sencillo y cómodo. Esto queda reflejado en el hecho de que los encuestados recomiendan extender este sistema a otras asignaturas y finalidades, tales como la revisión de exámenes.
\end{abstract}

Palabras clave: Sistema tutorial, tecnología de la información, innovación pedagógica, modernización.

\begin{abstract}
In this work we analyze the use of tools for planification and reservation of office hours, as well as the use of the videoconference tools in order to attend to these office hours. The study focuses on undergraduate students in the University of Córdoba during the term period 2019/2020. These tools allow students and professors a better organization of the tutorial action. Moreover, these tools became essential as consequence of the situation generated by COVID-19. This made necessary not only the virtualization of the office hours, but all the teaching as well. After the term period 2019/2020, during which this experience was held, students answered to an anonymous questionary about these tools. A very good success and good assessment of them was obtained. The conclusion was that not only they make easier to attend to office hours, but it is an easy-to-use system. This is a consequence of the fact that the students recommend extending this initiative to other subjects and aims, for instance, the revision of exams.
\end{abstract}

Key Words: Tutorial system, information technology, educational innovation, modernization.

\footnotetext{
I Universidad de Córdoba (España), to mas.morales@uco.es; CÓDIGO ORCID: 0000-0001-7162-9672

2 Universidad de Córdoba (España), alma.albujer@uco.es; CÓDIGO ORCID: 0000-0002-2384-9061

3 Universidad de Córdoba (España),jgarres@uco.es; CÓDIGO ORCID: 0000-0003-2013-6127

4 Universidad de Córdoba (España), jherrera@uco.es; CÓDIGO ORCID: 0000-0003-3038-5514
} 


\section{INTRODUCCIÓN}

La incorporación en el ámbito económico, social y académico de las tecnologías de la información y de la comunicación (TIC) ha permitido aumentar la calidad en todos los procesos. En el caso concreto de la docencia, la llegada de las TIC en el aula permite mejorar los procesos educativos tal y como demuestran las diversas propuestas que en este sentido se han llevado a cabo en los últimos años (véase Canay Pazos et al., 2016, 2018 y Blanco Medina et al., 2019). Sirvan como ejemplo las diferentes propuestas que en los últimos años se han planteado en la Universidad de Córdoba a través del Plan de Innovación y Buenas Prácticas docentes (véase UCO, n.d.). En diversos estudios(véase Grupo de trabajo de directores TI, 2018 entre otros), se aprecia cómo el alumnado universitario demanda un mayor uso de las herramientas TIC, a la vez que hace uso de ellas, empujando en el proceso de digitalización de la universidad hoy día. El papel de las TIC en el futuro de la enseñanza superior fue también estudiado en Telefónica (2012), donde destaca el carácter inmóvil de la universidad, que se enfrenta a un perfil de estudiante moderno, formado en la era digital. Precisamente, como se indica en Gisbert y Esteve (2011), las características de estos estudiantes pueden ser un puente hacia dicha digitalización de la universidad. Este proceso de digitalización en universidades británicas se estudia en PwC (2015), Newman y Beetham (2017) y Newman, Beetham y Knight (2018). Por otro lado, dentro del proceso educativo y muy especialmente en la educación superior, cobra especial relevancia la acción tutorial (Lapeña Pérez, Martínez Ruíz y Sauleda Parés, 2010, Gezuraga Amundarain y Malik Liévano, 2015, Montesinos Padilla, 2017, León Huertas, González López y Eslava Suanes, 2019). Los beneficios que ésta tiene para el rendimiento del alumnado son de gran importancia. Se observa cómo aquellos estudiantes que asisten asiduamente a tutorías son capaces de seguir mejor día a día la asignatura y sus resultados mejoran en las diferentes tareas de evaluación. Lamentablemente, el uso de la acción tutorial por parte del alumnado es en general escaso.

El análisis aquí presentado parte de un Proyecto de Innovación Docente llevado a cabo durante el curso 2019/2020 en el marco del Plan de Innovación y Buenas Prácticas Docentes de la Universidad de Córdoba (UCO, 2019, Implantación de herramientas TIC para la potenciación de la acción tutorial, 2009-1-5008, p. 3). En este proyecto se pretendió aplicar las TIC para la mejora y potenciación de la actividad de tutorización en las asignaturas de matemáticas de los diferentes grados. Para ello, se trabajó fundamentalmente en dos ámbitos:

En primer lugar, selección y uso de una aplicación móvil y plataforma web para una gestión más efectiva de las actividades de tutorización. Esta aplicación se puso a disposición del alumnado y del profesorado. Mediante ella, el alumnado dispone de un sistema de reserva de horarios de tutoría, facilitando su uso. Además, mediante la misma aplicación, el profesorado puede en todo momento gestionar dicha acción tutorial.

En segundo lugar, iniciar un proyecto piloto en el Departamento de Matemáticas de la UCO de uso de herramientas de virtualización, como Blackboard Collaborate, para la acción tutorial de asignaturas de matemáticas, facilitando su acceso al alumnado con dificultades de desplazamiento. Entiéndase como dificultades de desplazamiento no sólo el acceso al campus, sino incluso dentro del mismo, cuya estructura implica 
grandes desplazamientos entre aulas y despachos. Si además añadimos que la UCO cuenta con diferentes campus, dichas herramientas permiten, adicionalmente, que el profesorado con docencia en campus diferentes pueda atender convenientemente a su alumnado.

Paradójicamente, esta experiencia se propuso antes de que aconteciera la amenaza sanitaria que la COVID-19 ha constituido y sigue constituyendo. Esta situación excepcional obligó a empresas y administraciones públicas a rediseñar su estructura y procesos. La universidad no escapó a esta revolución digital que se ha venido imponiendo a marchas forzadas.

Especialmente durante el periodo de confinamiento y los meses que siguieron, la temática tratada por este Proyecto de Innovación resultó ser de máxima actualidad e importancia ante la imposibilidad de continuar con el método tradicional de trabajo. Las herramientas digitales se volvieron imprescindibles a lo largo del curso 2019/2020 a causa de la crisis de la COVID-19, ya que la práctica totalidad de las universidades públicas españolas se vio forzada a virtualizar su docencia. Las TIC pasaron de ser usadas de forma mayoritaria como una herramienta que aportaba un valor añadido, a convertirse repentinamente en un eje transversal del proceso enseñanza-aprendizaje. Sería en particular en este marco en el que el Proyecto de Innovación propuesto se desarrollaría plenamente y llegaría a adquirir una gran relevancia, continuando vigentes las estrategias desarrolladas a día de hoy, durante el curso 2020/2021.

El objetivo de este artículo es presentar y analizar una herramienta disponible online y a través de aplicaciones móviles para la organización y potenciación de la acción tutorial que fue utilizada durante el Proyecto de Innovación anteriormente citado (UCO, 2019, Implantación de herramientas TIC para la potenciación de la acción tutorial, 2009-1-5008, p. 3), así como presentar los resultados, todos muy positivos, que se obtuvieron en esta experiencia. Para ello, se presentarán las valoraciones y opiniones recabadas entre el alumnado mediante una encuestarealizada a estudiantes de la UCO que participaron en el Proyecto.

\section{DESARROLLO DE LA EXPERIENCIA DE INNOVACIÓN}

El desarrollo de la experiencia, dentro del marco del Proyecto de Innovación Docente propuesto, pretendió llevar a cabo una mejora docente a la actividad de tutorización del alumnado a través de la incorporación de las TIC. Para ello, se siguieron las etapas que se describen a continuación:

\subsection{Elección de una aplicación web y de una aplicación móvil}

Se valoraron diversas herramientas, buscando que incluyeran tanto aplicación web como móvil, que permitiesen:

- Al alumnado: poder consultar los horarios de tutoría disponibles, hacer reservas y poder gestionar mejor su tiempo y por tanto su rendimiento académico.

- Al profesorado: poder realizar una mejor gestión de la acción tutorial, facilitando su acceso por parte del alumnado, así como un tratamiento más personalizado.

El punto de partida fueron diversas plataformas existentes en internet que permiten, desde el punto de vista empresarial, gestionar un sistema de reservas o citas. 
El objetivo primero era pues adaptar esta herramienta al entorno académico. Entre las opciones que se barajaban se priorizaron las siguientes características:

- Debía tener acceso tanto web como desde aplicación móvil para facilitar su acceso y uso.

- Teníaque ser lo suficientementeconfigurable y adaptable al entorno académico.

- Se buscaron alternativas que dispusiesen de versiones gratuitas con la suficiente funcionalidad para poder utilizarlas sin necesidad de pago de una inscripción.

Tras varias valoraciones y deliberaciones, la opción elegida fue Setmore [véase Setmore (n.d.)]. Se trata de una herramienta diseñada con el objetivo, tal y como se anuncia en su web, de organizar un negocio con reservas automatizadas en línea las 24 horas del día, los 7 días de la semana, recordatorios, pagos y más. Esta herramienta verificaba todas las características antes planteadas $y$, aunque inicialmente estaba diseñada para negocios, era fácilmente adaptable para su uso en entornos académicos (véase el anexo 1).

\subsection{Formación del profesorado en el uso de las TIC}

Una vez elegida la plataforma para la reserva de horarios de tutoría, se procedió a la formación del profesorado participante en el Proyecto en el uso de la misma. Para ello se llevaron a cabo dos sesiones formativas en las que el profesorado procedió a la configuración de la aplicación y su adaptación particular para el uso en sus asignaturas.

Cabe destacar que la aplicación en sí es de fácil uso, sobre todo desde el punto de vista del alumnado, pero la configuración de la misma puede resultar algo más elaborada. Sin embargo, las acciones formativas llevadas a cabo dejaron claro que la consecución de este objetivo estaba al alcance del profesorado que participó y se elaboró el manual que se adjunta como anexo 1 a este artículo, resultando muy provechoso parael profesorado que, en un primer momento, no participó en el Proyecto, pero que posteriormente se adhirió al mismo dada su utilidad. De hecho, en dicho manual se presenta la forma en la que puede llevarse a cabo la configuración de la aplicación Setmore para adaptarla a la finalidad buscada en el Proyecto.

Junto con la formación en el uso de esta herramienta, se impulsaron herramientas TIC basadas en sistemas de videoconferencia para poder potenciar la tutorización, no solo de forma presencial, sino también online. Se introdujo principalmente la herramienta Blackboard Collaborate, la cual cuenta con soporte oficial en la UCO. La idea inicial era que, en el momento de la reserva a través de la aplicación de un horario concreto, el alumnado indicase si prefería que la tutoría fuese en modalidad online. En aquel entonces poco hacía presagiar que esta sería la única modalidad posible tras la situación excepcional y el periodo consiguiente de confinamiento a causa de la COVID-19.

\subsection{Difusión y potenciación de las herramientas disponibles entre el alumnado}

Como paso lógico siguiente, se procedió a difundir las herramientas disponibles entre el alumnado con el fin de motivar y fomentar su uso. Para ello, el profesorado participante destinó una franja horaria (aproximadamente una media hora) dentro de las diferentes asignaturas que impartía para presentar y mostrar, en vivo y en directo, las herramientas disponibles.

La acogida de estas herramientas por parte del alumnado fue muy positiva, tal y como se mostrará posteriormente en los resultados recogidos a través de una encuesta realizada entre el alumnado participante. 


\subsection{Difusión y coordinación de los recursos y herramientas disponibles}

Si bien la experiencia se inició con el profesorado participante en el Proyecto, 11 profesoras y profesores del Departamento de Matemáticas de la UCO, posteriormente se procedió a su difusión entre otro profesorado para que pudiesen hacer uso de la misma. Para ello, el manual desarrollado y adjunto como anexo 1 se puso a disposición del profesorado de la UCO.

La experiencia fue satisfactoria, si bien se hace patente la necesidad de dar una mayor difusión para llegar a un mayor público. Es por ello que el presente artículo contribuirá de forma decisiva a la difusión de esta experiencia y a los beneficios que puede tener la misma.

\subsection{Valoración de la experiencia}

Con el fin de valorar la experiencia llevada a cabo, se elaboró un cuestionario anónimo online para que el alumnado que había participado en esta experiencia piloto pudiese expresar su opinión sobre la misma.

Este cuestionario fue publicitado en las páginas Moodle de las asignaturas en las que el profesorado participante impartía docencia y estuvo disponible durante 15 días para recabar la mayor información posible. Los diferentes resultados se presentarán en la siguiente sección.

\section{RESULTADOS}

Durante el desarrollo del Proyecto la consecución de los objetivos inicialmente previstos resultó muy satisfactoria. En particular:

- Se ha implementado y hecho uso de una aplicación web y aplicación móvil que ha permitido:

- Al alumnado: optimizar su tiempo, y con ello su rendimiento académico, al poder consultar los horarios de tutoría disponibles y resenvar espacios para la acción tutorial.

- Al profesorado: mejorar la calidad de la acción tutorial, al poder realizar una mejor gestión de ella, facilitando su acceso por parte del alumnado, así como un tratamiento más personalizado.

- Se ha mejorado el acceso del alumnado a la acción tutorial, mediante la potenciación de la tutorización virtual individual y colectiva. Además, la formación del profesorado en herramientas online de tipo videoconferencia ha resultado de vital importancia durante el periodo de confinamiento.

- Se ha formado al profesorado en el uso de las TIC, con el fin de incorporarlas para el desarrollo de la acción tutorial.

- Se ha difundido y se ha coordinado el uso de los recursos inicialmente previstos, así como otros nuevos que se han incorporado durante el estado de alarma.

- Se ha valorado el uso de las herramientas desarrolladas por parte del alumnado mediante la elaboración de una encuesta online en la que se preguntaba por las diferentes cuestiones que se presentan a continuación y cuyos resultados se presentan con detalle. 


\section{Datos del alumnado: titulación y asignatura cursada, edad y sexo}

Contestaron la encuestaun total de 51 alumnos y alumnas. Es cierto que el número total es pequeño, pero cabe mencionar no obstante que el uso de la tutorización por parte del alumnado es baja en general. Precisamente el objetivo principal de la experiencia era fomentar el uso de la misma. El rango de edad variaba entre los 18 y 28 años, siendo el $68,6 \%$ de los encuestados y encuestadas de una edad inferior o igual a 21 años. El $56,9 \%$ son mujeres y el $43,1 \%$ hombres.

Teniendo en cuenta que el profesorado participante es miembro del Departamento de Matemáticas, los grados y asignaturas que cursaba el alumnado corresponden a carreras de ciencia e ingeniería. La distribución es bastante heterogénea con participantes de los grados de ingeniería electrónica, eléctrica, mecánica, informática, grado en biología y en ciencias ambientales. Las asignaturas cursadas corresponden a materias relacionadas con las matemáticas impartidas en su mayoría en los primeros cursos de esas titulaciones.

\section{Frecuencia de uso de las tutorías en la asignatura}

Se preguntó al alumnado que valorara el uso que hace de la tutorización en una escala del 1 al 5 , siendo 1 poco frecuente y 5 muy frecuente. En general, y como puede deducirse de la figura 1, el alumnado hace un uso poco frecuente de las tutorías. De hecho, un $64,8 \%$ de los encuestados y encuestadas consideran que su asistencia a tutorías es entre muy poco o poco frecuente (puntuación 1 ó 2). Los motivos de esto son variados, pero reflejan la necesidad de considerar nuevas vías (como la presentada en esta experiencia) que incentiven entre el alumnado el uso de tutorías (ya sean individuales o grupales).

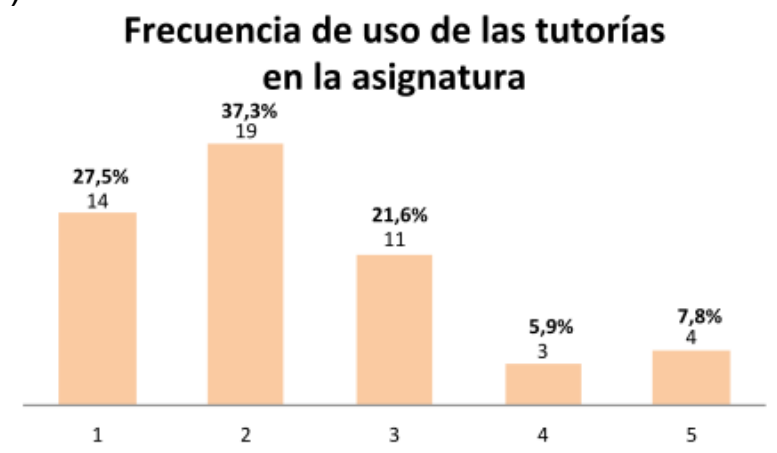

Figura 1. Frecuencias de uso de las tutorías en la asignatura.

Fuente: Elaboración propia.

Uso del sistema de reserva online para tutorías y mejora del proceso de tutorización. Se preguntó en qué proporción se había hecho uso del sistema de reservas, así como si consideraban que ese sistema mejora y facilita el uso y asistencia a las tutorías.

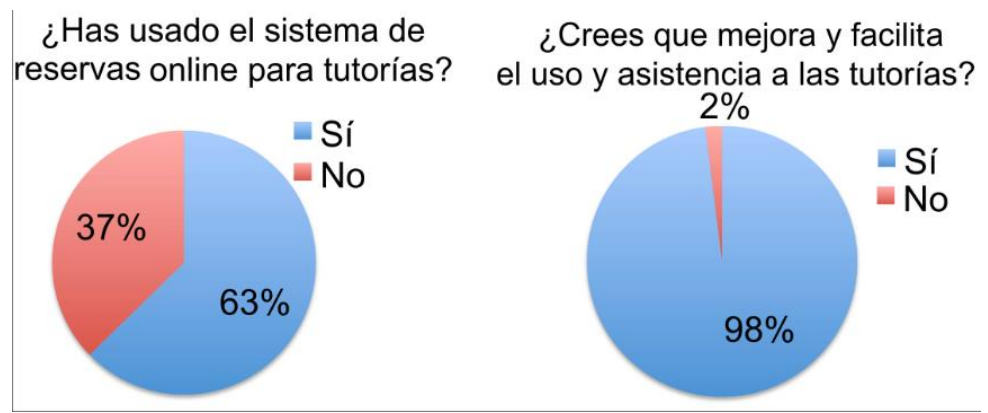

Figura 2. Respuestas del estudiantado a preguntas sobre la utilidad del sistema de reservas y su facilidad de uso.

Fuente: Elaboración propia. 
En la figura2 (izquierda) se muestra que en esta experiencia más de la mitad del alumnado encuestado ha hecho uso de la herramienta de reservas, lo cual refleja una tendencia positiva a un mayor uso de tutorías si lo comparamos con lo obtenido en la figura 1. Además, como podemos ver en la misma figura 2 (derecha), la respuesta del alumnado ha sido mayoritariamente positiva respecto al uso de dicha plataforma, entendiendo que su uso mejora y facilita la asistencia a tutorías.

\section{Valoración de la herramienta de reserva}

Con el fin de valorar la utilidad de la herramienta y del proceso de reserva de tutoría, se pidió que se valoraran en una escala del 1 al 5 los siguientes ítems (siendo 1 valoración muy negativa y 5 valoración muy positiva):

- Facilidad y comodidad de uso del sistema de reservas: 89,8\% la valora de forma muy positiva (puntuación 4 o 5).

- Cómo afecta el sistema de reservas de tutoría a la organización del tiempo: $81,7 \%$ lo valora de forma muy positiva (puntuación 4 o 5).

- Valoración global del sistema de reservas para horario de tutorías: 80,4\% la valora de forma muy positiva (puntuación 4 o 5).

Estos resultados pueden verse en la figura 3.

Valoración de la facilidad y comodidad de uso del sistema de reservas

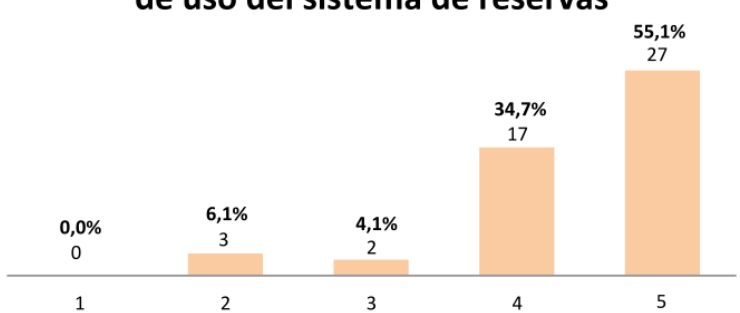

Valora de forma global el sistema de reservas de tutorías

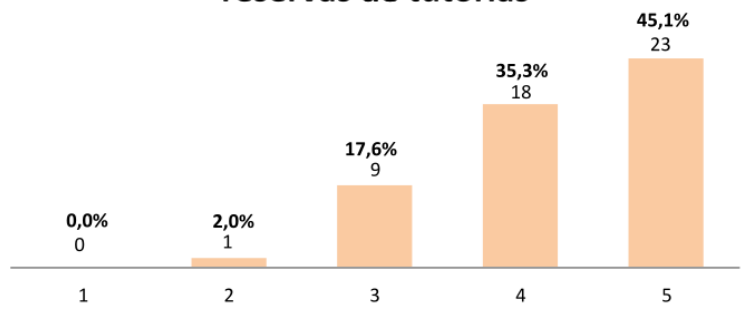

Figura 3. Valoración del estudiantado sobre la facilidad de uso del sistema de reservas y su impacto sobre su propia organización y una valoración global del mismo.

Fuente: Elaboración propia.

Puede concluirse por tanto que la herramienta escogida para el sistema de reservas ha sido adecuada, con una valoración muy positiva entre un porcentaje superior al $80 \%$ de los alumnos encuestados.

\section{Posibilidad de exportar la experiencia a otras asignaturas y procesos:}

- Finalmente, con el fin de valorar hasta qué punto la experiencia es exportable, se plantearon dos cuestiones (véase figura 4): En la primera (figura 4, izquierda), se preguntaba al alumnado si pensaban que fuera posible expandir la experienciaa otras asignaturas. El resultad o fue claramente positivo, con un $80 \%$ 
del alumnado encuestado mostrando interés en el uso de la herramienta en otras disciplinas (puntuación 4 o 5).

- La respuesta también fue positiva al preguntar si consideraban la herramienta de utilidad para la organización de la revisión de exámenes (figura 4, derecha), aunque en este caso no se alcanzó una aceptación tan alta como en la cuestión anterior (un 64\% la consideran muy útil con una puntuación 4 o 5).

¿Debería implantarse este sistema en otras asignaturas?

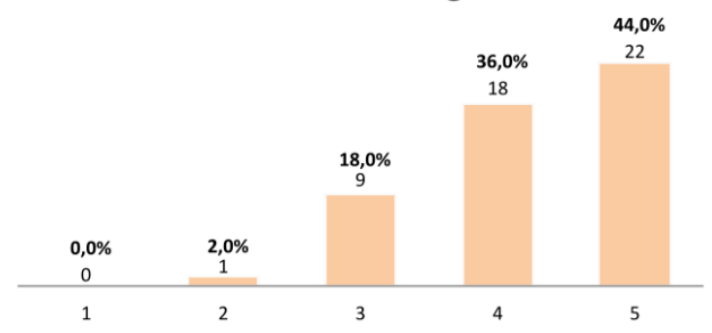

¿Es útil esta herramienta para organizar la revisión de exámenes?

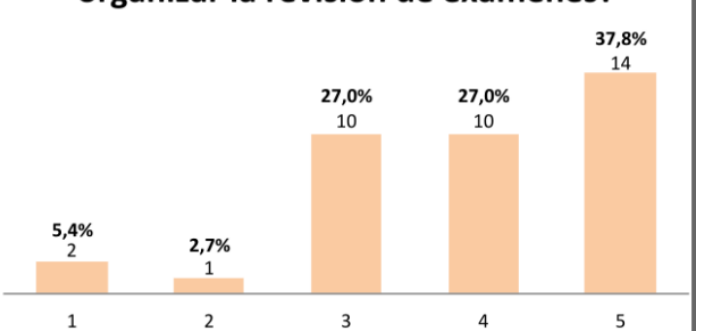

Figura 4. Valoración del estudiantado sobre si el sistema de reservas debería extenderse a otras asignaturas y a la organización de revisión de exámenes.

Fuente: Elaboración propia.

\section{CONCLUSIONES}

En el desarrollo de este proyecto queda demostrado que el uso de plataformas que permiten realizar de una forma dinámica la reserva de horario de tutorías por parte del alumnado tiene un impacto positivo, tanto sobre la organización de su tiempo, como sobre el acceso a las tutorías. En este proyecto se ha optado por la aplicación Setmore, cuyo uso ha sido satisfactorio tanto por parte del alumnado encuestado como por el profesorado que ha formado parte de dicho proyecto.

Los datos recabados sobre la acción tutorial reflejan que el estudiantado no hace un uso elevado de estas tutorías, probablemente por la dificultad añadida de organización y planificación. Se espera por tanto que la flexibilidad que dan tanto el sistema de reserva de tutorías como la opción de realizar tutorías (individuales o grupales) de forma virtual, impulse el uso de las acciones tutoriales que tan útiles son para el aprendizaje del alumnado. Así se deduce de los resultados obtenidos en esta experiencia, en donde casi la totalidad del alumnado opina que la iniciativa planteada mejora y facilita la acción tutorial.

A la vista de la encuesta realizada, el alumnado valora de forma muy positiva el uso de herramientas de reserva de tutorías. Piensan que la herramienta es cómoda y fácil de usar, mejorando la organización y planificación de su tiempo. Incluso indica que estas deberían extenderse a otras asignaturas y escenarios, como la revisión de exámenes.

Hemos de concluir por tanto que la experienciaha resultado provechosa, no solo para el alumnado, sino también para el profesorado. Los resultados del Proyecto de Innovación desarrollado han mejorado e incentivado el uso de la acción tutorial. Las herramientas utilizadas y desarrolladas han tenido muy buena acogida entre la comunidad universitaria participante en la experiencia.

\section{REFERENCIAS}

Blanco Medina, I., Cáceres Taladriz, C., Camarillo Casado, J., Esteban Sánchez, N., Martínez, J.A., Nuere Salgado, L., Sota Equizábal, J. M., Turró Ribalta, C., \& 
Villalonga Gómez, C. (2019). Informe de situación de las tecnologías educativas en las Universidades Españolas 2018. CRUE, Universidades Españolas. Recuperado de: https://tic.crue.org/wp-content/uploads/2020/09/TecnologiasEducativas-2018_DIGITAL.pdf

Canay Pazos, J. R., Llorens, F., Fernández-López, S., Rodeiro-Pazos, D., Ruzo, E., Martínez, A., Lainz, F., \& García-Peñalvo, F. (2018). UNIVERSITIC 2017. Análisis de las TIC en las Universidades Españolas. Recuperado de: https://tic.crue.org/publicaciones/informe-universitic-2017/

Canay Pazos, J. R., Llorens, F., Martínez, A., Fernández-López, S., Rodeiro-Pazos, D., Ruzo, E., Lainz, F., Molina-Carmona, R., Andreu Navarro, V., \& Zapata-Ros, M. (2016). UNIVERSITIC 2016: Análisis de las TIC en las Universidades Españolas. Recuperado de: https://tic.crue.org/publicaciones/informe-universitic-2016/

Gezuraga Amundarain, M., \& Malik Liévano, B. (2015). Orientación y acción tutorial en la universidad: aportes desde el aprendizaje-servicio. REOP - Revista Española de Orientación y Psicopedagogía, 26(2), 8-25. Recuperado de: http://revistas.uned.es/index.php/reop/article/view/15213

Gisbert, M., \& Esteve, F. (2011). Digital learners: la competencia digital de los estudiantes universitarios. La Cuestión Universitaria, 7, 48-59. Recuperado de: http://polired.upm.es/index.php/lacuestionuniversitaria/article/view/3359

Grupo de trabajo de directores TI. (2018). TIC 360: Transformación Digital en la Universidad. Crue Universidades Españolas, Madrid. Recuperado de: https://tic.crue.org/wp-content/uploads/2016/03/transformacion-digital-univ.pdf

Lapeña Pérez, C., Martínez Ruíz, M. A., \& Sauleda Parés, N (2010). Innovación en la acción tutorial: Análisis de caso en la universidad de Alicante. Revista de Educación $y$ Derecho., $1 . \quad$ Recuperado de: https://revistes.ub.edu/index.php/RED/article/view/2230

León Huertas, C., González López, I., \& Eslava Suanes, M. D. (2019). La tutoría como estrategia de trabajo compartido. Revista de Innovación y Buenas Prácticas Docentes, 8(2), 23-33. Recuperado de: https://www.uco.es/ucopress/ojs/index.php/ripadoc/article/view/12164

Montesinos Padilla, C. (2017). La acción tutorial como herramienta de aprendizaje. Especial referencia a la tutorización del alumnado con necesidades especiales. DOCENCIA Y DERECHO, 11. Recuperado de: https://www.uco.es/docencia_derecho/index.php/reduca/article/view/113

Newman, T., \& Beetham, H. (2017). Student digital experience tracker 2017: the voice of 22,000 UK learners. Recuperado de: https://repository.jisc.ac.uk/6662/

Newman, T., Beetham, H., \& Knight, S. (2018). Digital experience insights survey 2018: findings from students in UK further and higher education. Recuperado de: https://repository.jisc.ac.uk/6967/

PwC. (2015). The 2018 digital university: Staying relevant in the digital age. Recuperado de: $\quad$ https://www.pwc.co.uk/assets/pdf/the-2018-digital-university-stayingrelevant-in-the-digital-age.pdf.

Setmore (n.d.) Recuperado el 10 de diciembre de 2020, de https://www.setmore.com/es

Telefónica. (2012). Universidad 2020: Papel de las TIC en el nuevo entorno socioeconómico. Recuperado de: https://www.fundaciontelefonica.com/culturadigital/publicaciones/153/.

UCO (n.d.) Recuperado el 10 de diciembre de 2020, de http://www.uco.es/organizacion/calidad/innovacioneducativa/es/innovaciondocente 
UCO (2019) Resolución definitiva de la Modalidad 1: Proyectos de Innovación Docente. Curso 2019/2020 Recuperado de https://sede.uco.es/bouco/bandejaAnuncios/BOUCO/2019/00660\# 


\section{ANEXO 1. MANUAL DEL PROFESOR PARA SETMORE: CREACIÓN DE CUENTAS, CONFIGURACIÓN Y USO}

Con el fin de complementar lo expuesto en este artículo y permitir a cualquier docente aplicarlo en su propio entorno, se presenta en este anexo un manual en el que se dan las pautas básicas para que el profesorado pueda crear una cuenta en la aplicación Setmore y configurarla para su uso como un programa de gestión de la acción tutorial desde la aplicación web.

\section{Registro en Setmore}

El primer paso es el registro individual y la creación de una cuenta gratuita en la página web de la aplicación (Setmore, n.d.) siguiendo las instrucciones de la propia página.

\section{Configuración básica de la cuenta}

La configuración básica de la cuenta consta de cuatro pantallas en las que se solicitan los datos básicos del registro y que el usuario debe completar tras su registro:

- Tras entrar en la aplicación con la cuenta personal aparece la pantalla de bienvenida de la figura 5, en la que el usuario debe introducir sus datos. Aunque el inicio de la configuración puede aparecer en inglés, más adelante es posible cambiar el idioma y elegir, por ejemplo, el idioma español. No obstante, lo habitual es que en el formulario inicial aparezca el mismo idioma con el que se accedió a la página principal. Se sugiere como nombre del negocio Acción Tutorial - Nombre del usuario. En el tipo de industria se puede escoger Institución académica / Educational Institution.

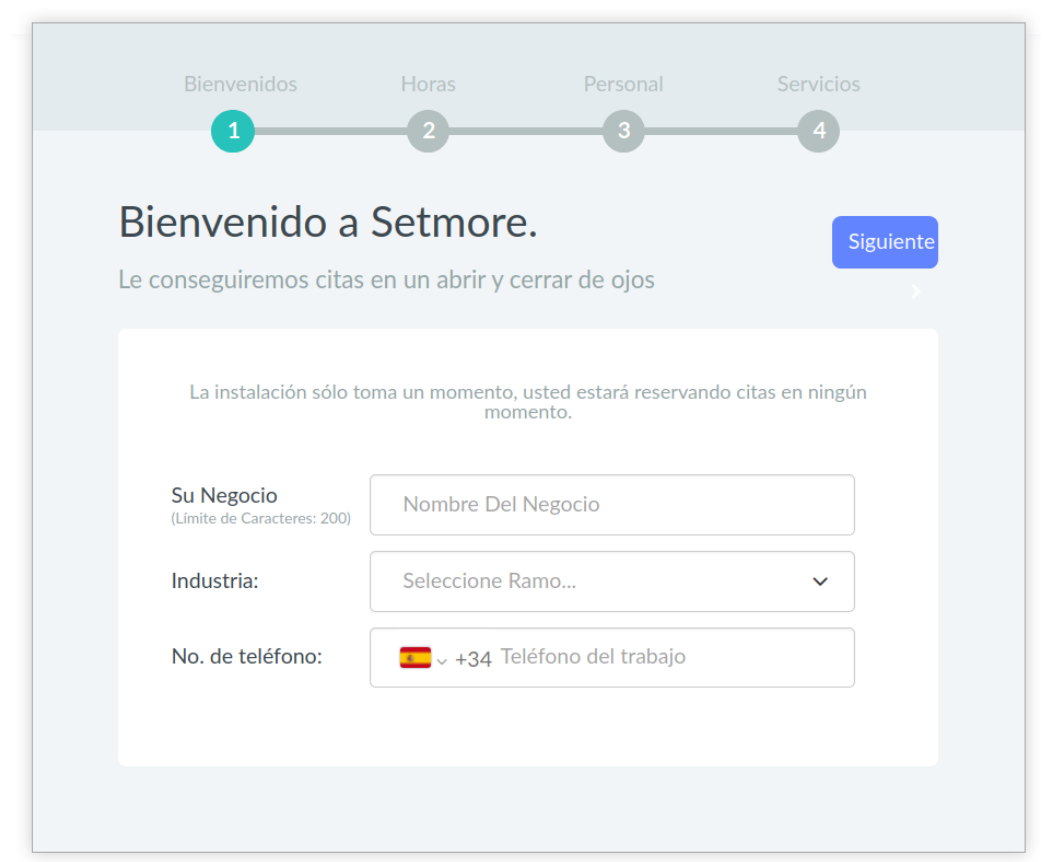

Figura 5. Pantalla de bienvenida.

Fuente: Setmore (n.d.). 
- En la pantalla siguiente el usuario debe indicar los días de la semana y horas de trabajo. Hay que tener en cuenta que estas horas no tienen por qué coincidir con las que el usuario vaya a dedicar a la acción tutorial. Por lo general será un horario más amplio, dentro del cual se podrá escoger, más adelante, las horas reservadas para tutorías. La idea es que este horario de trabajo sea fijo (por ejemplo, de lunes a viernes de 8:30 a 17:00) y en dicho intervalo se podrán establecer los horarios de tutoría, que se podrán modificar cuando sea necesario con facilidad.

- A continuación, viene la configuración del personal, que se debe dejar por defecto puesto que ya aparecerá el usuario con el nombre y correo electrónico con el que haya creado la cuenta.

- El último paso en la configuración básica es el de seleccionar los servicios. Estos deben corresponderse con las duraciones estimadas de las tutorías. Se pueden poner varios servicios como podrían ser tutorías de 5 minutos, 15 minutos etc. 0 lo que el usuario considere conveniente. El precio de cada servicio será de cero euros. Se muestra en la figura 6 un ejemplo con un único servicio.

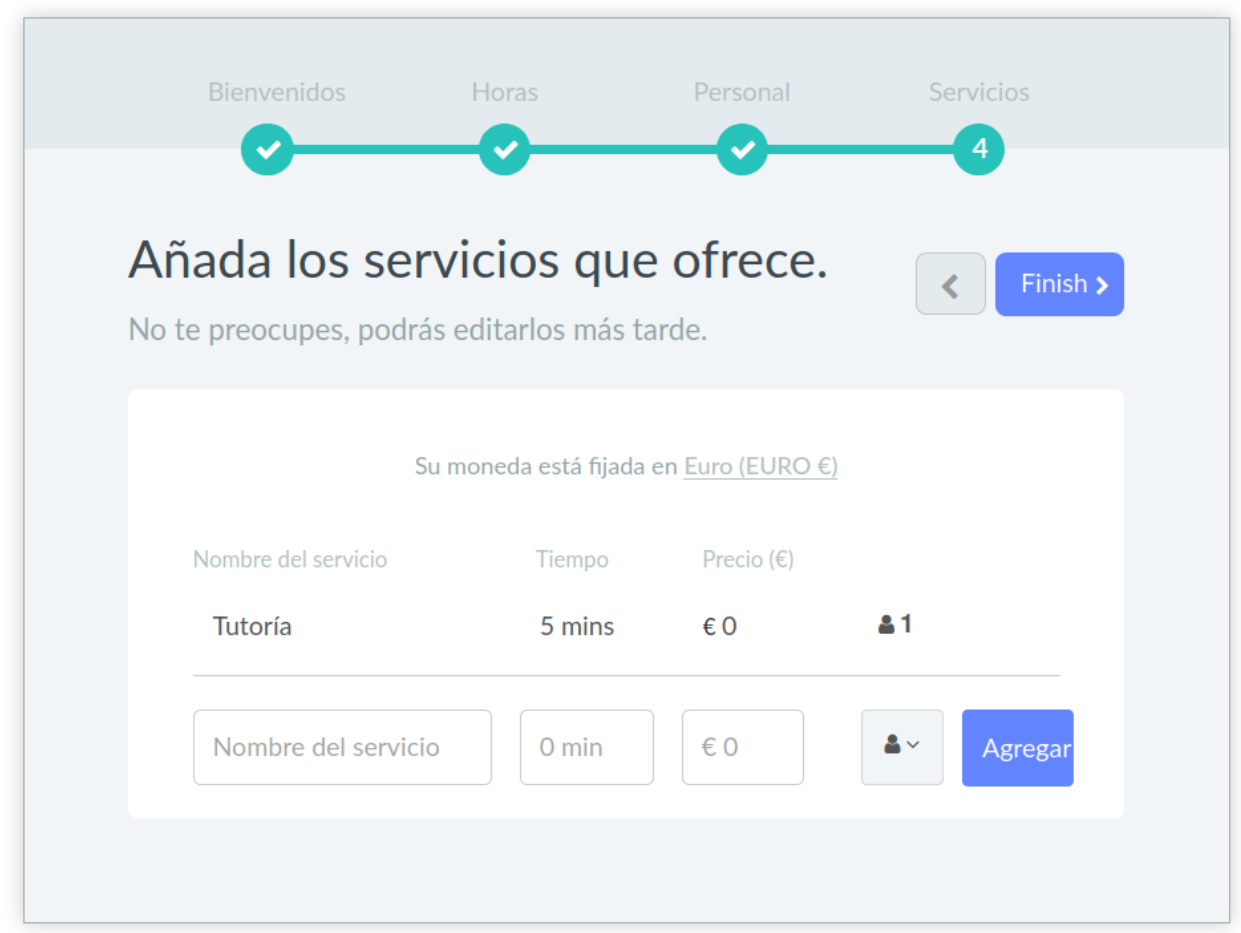

Figura 6. Servicios ofertados.

Fuente: Setmore (n.d.).

\section{Personalización básica de la cuenta}

Una vez realizada la configuración básica hay ciertos ajustes que el usuario puede y debe realizar para asegurar la correcta funcionalidad de la cuenta para el uso que se desea. Se detallan a continuación: 
- Si el idioma por defecto no es el preferido, se puede modificar. Para cambiar el idioma se debe seguir el camino Ajustes - Cuenta - Preferencias - Lenguaje Preferido (Settings - Account - Preferences - Preferred Language si lo tenemos en inglés). En este manual se elige el idioma español por defecto, por lo que, a partir de ahora, todas las opciones de configuración de este manual se referirán a este idioma.

- También en el anterior menú de Preferencias se pueden modificar ciertos aspectos como el tipo de calendario: semanal o mensual, y se pueden desactivar las estadísticas de citas.

- Los detalles que se verán en la página de reservas tales como el título, el logo (opcional) etc. se completan siguiendo el camino Ajustes - Cuenta-Detalles de la compañía. Además, en esta pestaña observamos el campo de la URL de la página de reservas. Esta será la dirección que se debaproporcionar al alumnado para la reserva de tutorías.

- En Ajustes - Personal - Horas Laborales se se debe indicar el horario de tutoría, tal y como puede observarse en la figura 7 . El rango de tutorías debe estar comprendido en el horario de trabajo establecido en la configuración básica. Es importante tener en cuenta que sólo se permite poner un intervalo reservado para la acción tutorial al día. En caso de querer poner dos intervalos distintos en el mismo día se introducirá como hora de inicio el inicio del primero y como hora de finalización el final del último. Más adelante podremos separar dichos intervalos.

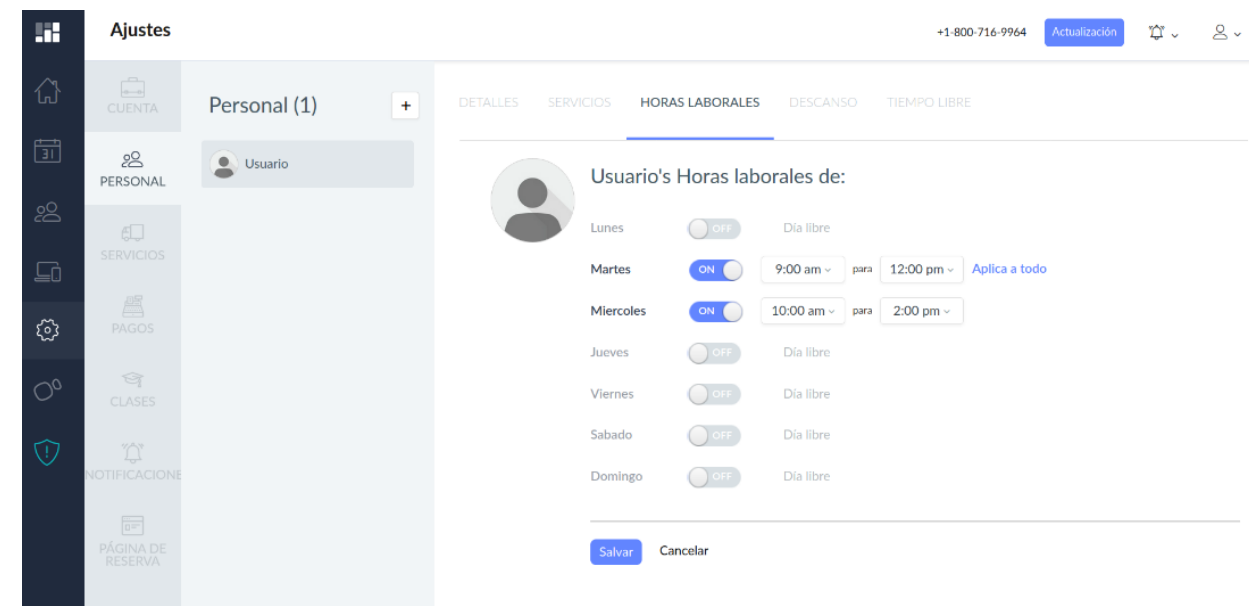

Figura 7. Horas laborables.

Fuente: Setmore (n.d.).

- En Ajustes - Página de Reserva-Política de Reservación se configuran las opciones que el alumnado deberá introducir en la reserva:

- Las Políticas de Nombramiento (tiempo de espera de la cita, tamaño de la ranura, ventana de programación y política de cancelación) se ajustarán según las preferencias de cada usuario.

- En Servicios de Clase el usuario debe asegurarse que quede desactivada la pestaña Precios de servicio/clase. 
- En Flujo de Reservas el usuario debe activar la pestaña Saltar el personal y desactivar Inicio de sesión del cliente, siendo recomendable desactivar el resto de las pestañas.

- En Cómo los clientes reservan sus propias citas en líneael usuario puede escoger los campos que queramos que el alumnado introduzca al realizar una reserva como por ejemplo Email, Titulación o Motivo de la tutoría, pudiendo elegir que estos campos sean o no obligatorios como puede observarse en la figura 8.
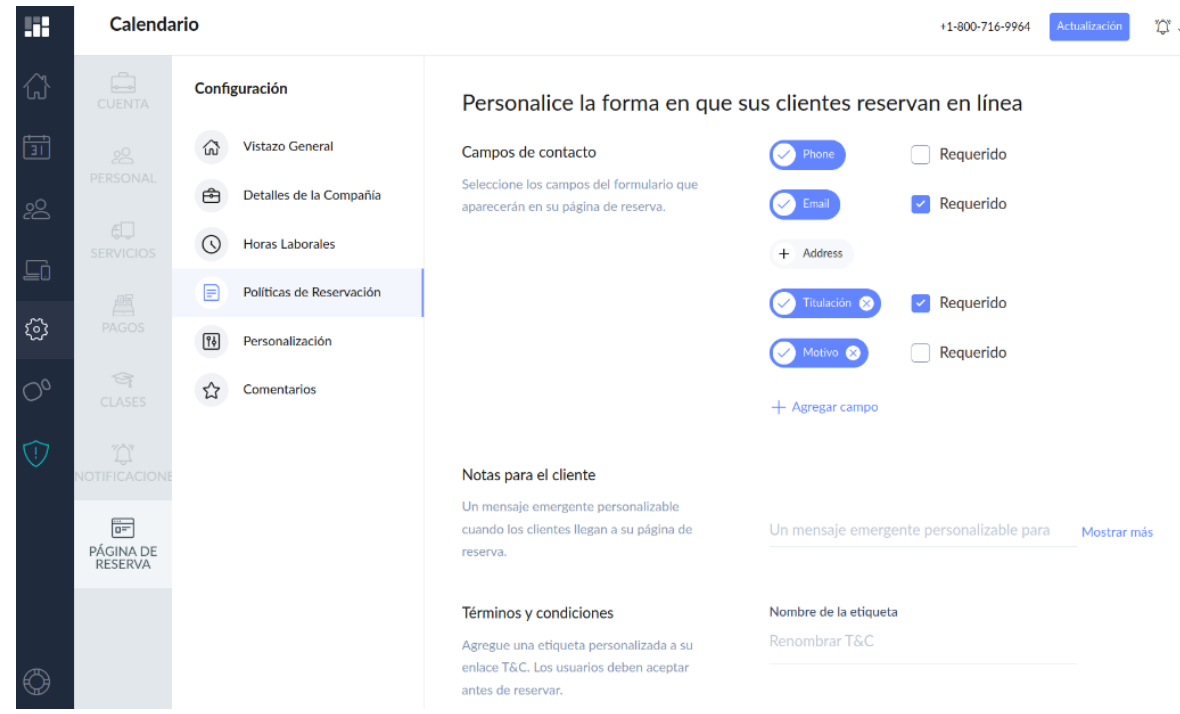

Figura 8. Campos requeridos en la reserva.

Fuente: Setmore (n.d.).

- La ventana Ajustes - Página de reserva-Personalización se ajustará según la figura 9. Además, en el campo Mostrar al proveedor como, el usuario deberá indicar Profesor o Profesora responsable de las tutorías.
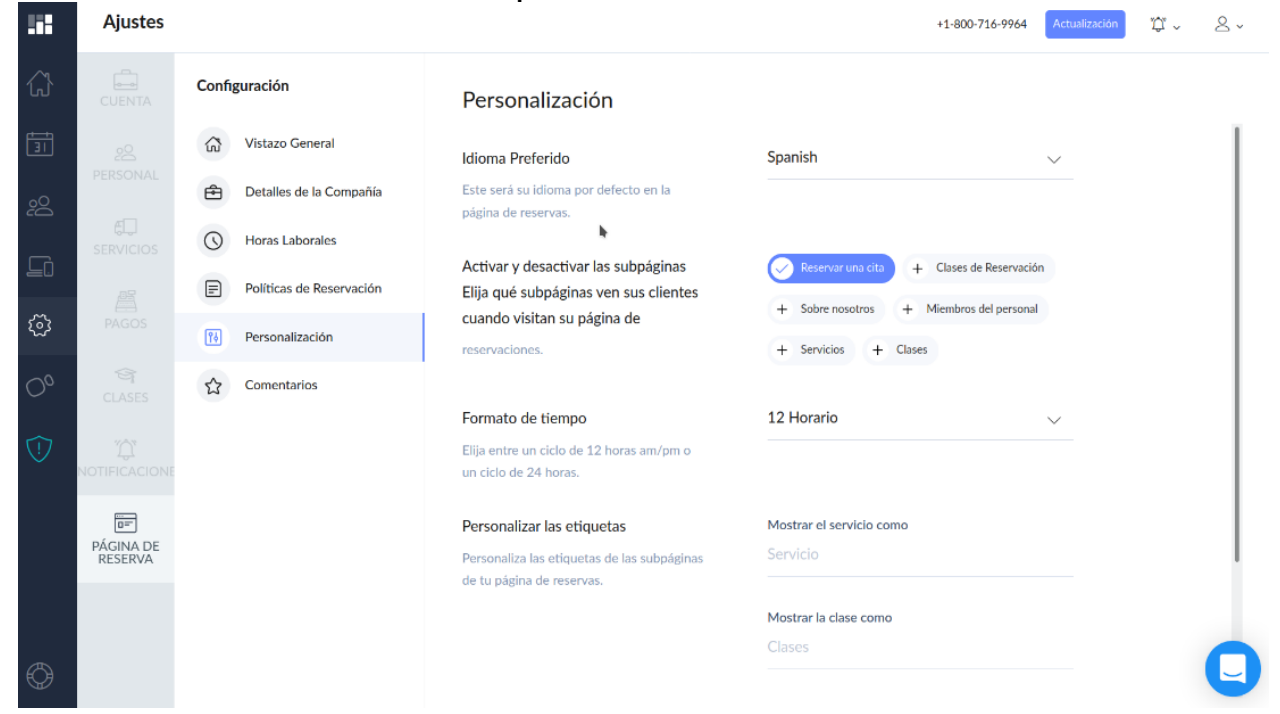

Figura 9. Personalización de la cuenta.

Fuente: Setmore (n.d.).

Con estas indicaciones damos por terminada la configuración básica para que el alumnado pueda hacer reservas. Podemos hacer una prueba en la URL que figura en Ajustes - Cuenta - Detalles de la compañía. 


\section{Personalización específica del docente}

Las opciones del docente se configuran en Ajustes - Personal. Aquí se encuentrael perfil del docente con las siguientes pestañas:

- Detalles: el usuario puede completar su descripción y sus preferencias, de acuerdo a la información que se mostrará luego en la página de reservas.

- Servicios: se deben seleccionar todos los servicios que hemos configurado.

- Horas laborales: deben coincidir con el horario destinado por el profesorado a la acción tutorial. Si este cambia, es aquí donde debemos modificarlo.

- Descanso: permite establecer un periodo en el que no se estará disponible para las tutorías. Esta opción sirve, por ejemplo, para crear dos intervalos distintos de tutorías en el mismo día. Así, supongamos que los miércoles se quiere establecer tutorías en las franjas de 10:00 a 12:00 y de 13:00 a 14:00. La solución sería escoger como horario laboral la franja de 10:00 a 14:00 y añadir un Descanso ese día de 12:00 a 13:00.

- Tiempo libre: se utiliza para definir si en algún momento el docente no va a estar disponible para atender al alumnado, como puede ser por asistencia a un congreso, una reunión laboral, periodo de vacaciones, etc.

- En Ajustes - Servicios el usuario puede modificar, eliminar o crear nuevos servicios con respecto a los ofertados al inicio, que normalmente se corresponden con opciones de reservas de distinta duración.

Por último, se pueden configurar las opciones de avisos de reservas en Ajustes - Notificaciones:

- En la pestaña Clientes se recomienda activar la casilla Correo Electrónico como medio de notificación de las próximas citas a los clientes, y configurar los avisos que le llegan al alumnado según los ajustes de la figura 10. 

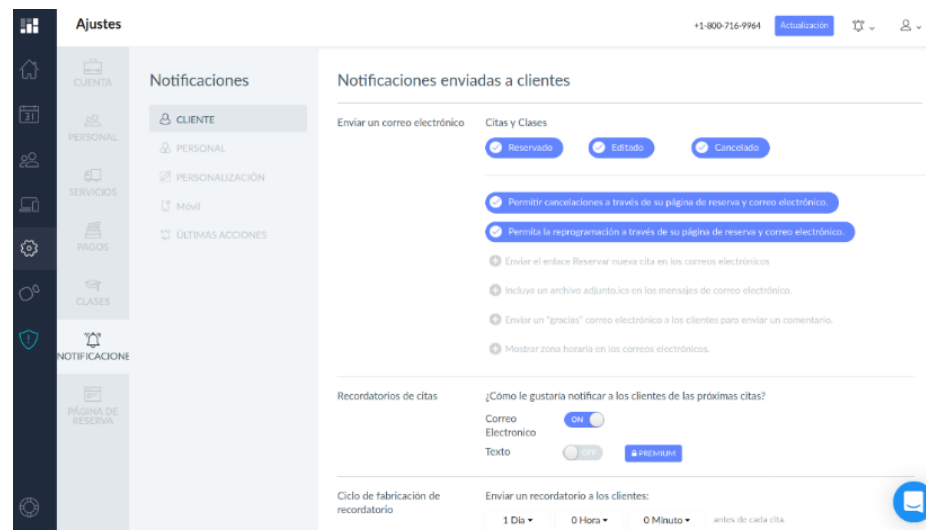

Figura 10. Configuración de las notificaciones enviadas a clientes.

Fuente: Setmore (n.d.).

- En la pestaña Personal se configuran los avisos que le llegarán al docente. Se recomienda activar la casilla Correo Electrónico como vía de notificación de las próximas citas y la configuración mostrada en la figura 11.

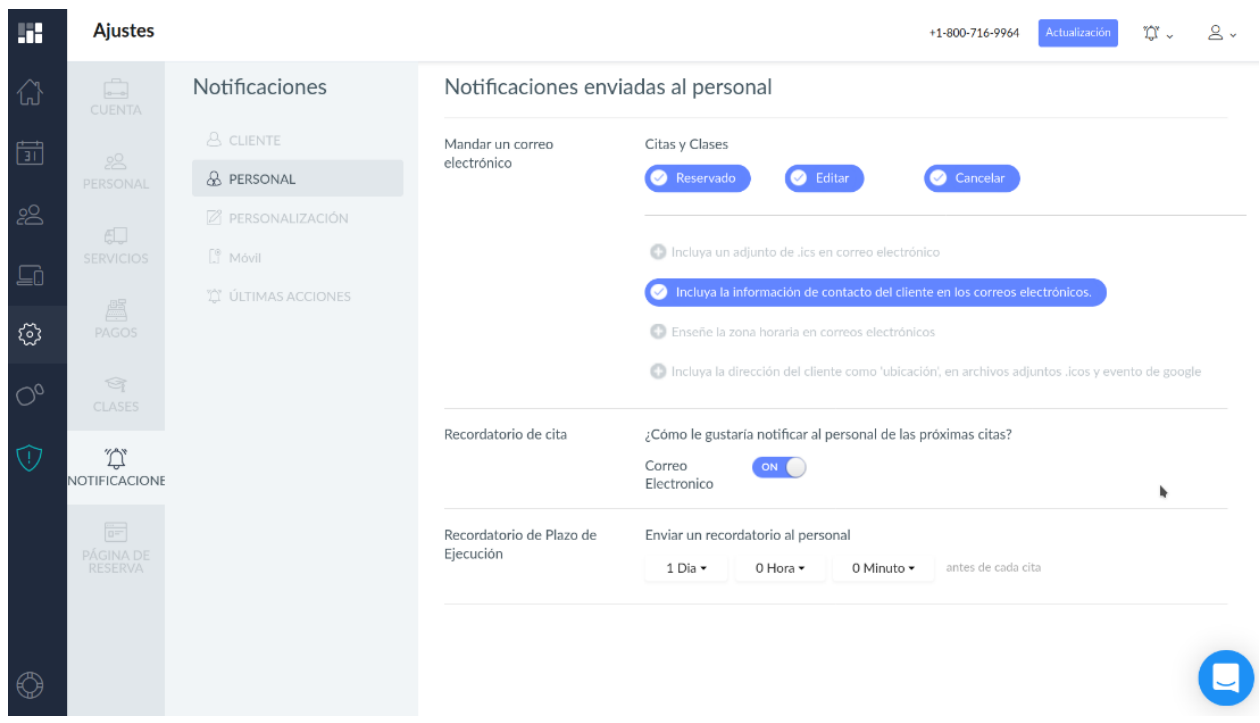

Figura 11. Configuración de las notificaciones enviadas al personal.

Fuente: Setmore (n.d.).

La aplicación Setmore tiene su propia aplicación gratuita para dispositivos móviles, bien sea para un sistema operativo Android como para iOS. Una vez que el usuario se descargue la aplicación en su dispositivo móvil e inicie sesión con las credenciales con las que se registró desde el navegador, la configuración realizada para su cuenta desde el navegador se exportará de forma automática a la aplicación del dispositivo móvil. A partir de ese momento el usuario puede llevar a cabo la gestión de su acción tutorial por el medio que considere más oportuno. 